\title{
DESIGN AND AUTOMATION OF INDUSTRIAL MACHINE USING ESP8266 MODULE
}

\author{
Sanket N. Gore ${ }^{1}$, Apeksha Sakhare ${ }^{2}$ \\ ${ }^{I}$ M.E, Student, Department of Computer Science \& Engineering, G.H.Raisoni College of Engineering, Nagpur, India \\ ${ }^{2}$ Assistant Professor, Department of Computer Science \& Engineering, G.H.Raisoni College of Engineering, Nagpur, \\ India
}

\begin{abstract}
Maintenance, Reliability of system are major issues in industry and having a time base machine can help to prevent the life, reliability of machine. This paper seeks to design and automatic real-time data entry and control system. The project is based on industrial purpose for the operation of industrial machine and also based on Time which is used to update the status of machine parts. Status of every spare part of machine is required to maintain the life of machine. The project comprises of Internet of Things which is interfaced with Hardware of machine through ESP8266 wifi module. Wifi module is used to send the status as well as data of machine on server in which hardware contain one Relay output for and two inputs from machine. The main goal of project is to reduce the errors of spare part calculation for machine maintenance.
\end{abstract}

Keywords: Internet of Things, ATmega Controller, ESP8266 wifi Module, Websocket(Server)

$* * *$

\section{INTRODUCTION}

The project comprises of server section and hardware section. Hardware section contain WIFI module which have a complete AT command library which is used for communication. This WIFI module is integrated with ATmega128 controller. Communication between WIFI module and controller is operating on serial communication. UART is a used to transmit and receive the commands to and from WIFI module and controller. Server is remote control for this project. Means Hardware will get each and every commands from server. To communicate with server, WIFI module need to ping to server for connection with server IP and port. Due to this connection, system will work as bidirectional. When hardware will ping to server then server will respond with the necessary data and depending on that data hardware will perform further operation.

\subsection{ATmega 128 Controller}

Microcontrollers are used in different systems such as remote control systems, power tools, home appliances, toys. By reducing the size and cost of controller compared to another devices which have a separate microprocessor, memory, and input/output devices. Microcontrollers makes it digitally control. The work investigates and implements a microcontroller-based adjustable system for a energy. Controller send as well as retrieve the data from and to the ESP8266 WIFI module.

\section{Features Atmega128}

128 Kbytes Flash Memory Program, 4Kbytes EEPROM, 4Kbytes Internal SRAM, 8-bit Timer/Counters(Two), Two Expanded 16-bit Counters/Timer, 10-bit ADC, 8-channel, Master/Slave SPI Serial Interface, Two Programmable
Serial USARTs, Two-wire Serial Interface, 53 Programmable I/O Lines, Seven interrupt source.

In Hardware connection UART(RXD/TXD) is used to interface ESP8266 wifi module. Two inputs are interfaced with interrupt and Relay output on controller i/o pin. Hardware also interfaced with RTC which is used to store current date and time for task.

\subsection{Power Supply}

Transformer: The transformer is used step down the power supply voltage from $0-230 \mathrm{~V}$ to $0-24$ Volt. If the secondary side has less number of coils than the primary side, the secondary side coil's voltage will decrease and the current will increase or decreased. This system is known as STEPDOWN transformer.

Step Down Transformer: Transformers are static devices which is used to convert electrical energy from one circuit to another circuit without changing frequency and power. Step down transformer means a system which reduces the supply voltage to the expected value. In our project we need 24 volt AC supply, therefore in this project $240 \mathrm{v}$ to $24 \mathrm{v}, 1 \mathrm{~A}$ transformer is used.

Rectifier Circuit: Rectifier is a circuit which is used to convert the AC supply into DC supply. For operating controller in this project we need regulated DC supply. Full wave rectifier circuit have capability of converting sinusoidal input signal into a unidirectional output signal.

Filter Circuit: The output of the rectifier is not pure DC, because it contain some amount of AC component which is called as ripple factor which gives the fluctuation. To minimize the ripple factor in the output of signal the filter 
circuit is used and circuit is connected after the rectifier circuit. In our project capacitor input filter is used. The capacitor is connected in parallel which is used to minimizethe ripple factor.

Regulator Circuit: In this project for the operation of IC we need +5 volt regulated supply for which a necessary voltage regulator circuit is used. A voltage regulator is a circuit that gives constant voltages without change in the load current. IC voltage regulators are versatile and generally used. The series consist regulator IC contain three terminal positive voltage regulators. These ICs are designed for fixed voltage and additional heat sink. These devices are not depending on external component.

\subsection{Industrial Machine}

Industries contain different machine for weight lifting. A simple example of machine is Hoist. Hoist is a machine which is used to lift weights from one place to another. Hoist contain different spare parts. This spare parts run equally from starting to end. Hoist contain spare parts like chain, motor, gears etc. which have their life to run. All this parameter runs till their life and need to be replaced with new one. If this parts are not get replaced with new one they will affect on other. Due to this, other parts will also get damaged.

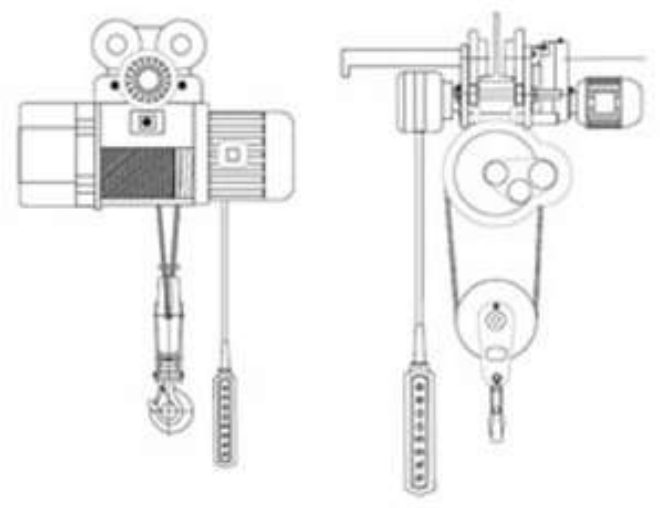

Fig -1: A Hoist Machine

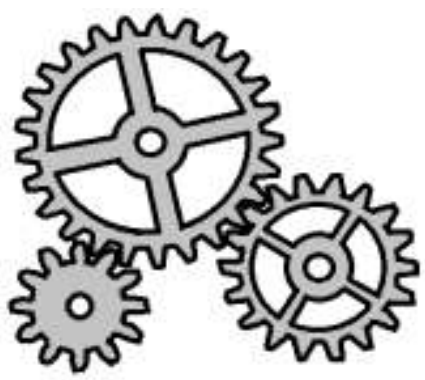

Fig -2: Gear (Combination of three)

According to this three gears, a least countof gear will be calculated and it will be given to the hardware first. Least count is calculated on the basis of life of gear. Similarly, for motor and chain least count will be calculated. UP and DOWN buttons are used to operate hoist system and count for spare parts will start increasing.

\subsection{ESP8266 Wifi Module}

The ESP8266 is low cost wireless module with a complete AT command library. This allows for easy integration with WIFI network through wireless communication. ESP8266 explored two modes: Station mode and Access point mode. As a station, the system connects to wireless network and reads data from internet. As access point mode, the ESP holds a small html website. ESP8266 works on 3.3 volts.

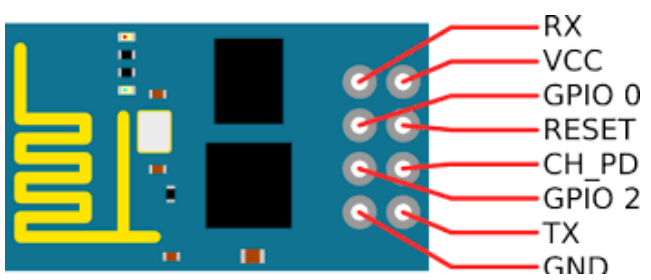

Fig -3: ESP8266 Pinout

- $\quad \mathrm{RX} / \mathrm{TX}$ - UART Communication pins

- VCC - Power Supply, device works on 3.3V supply.

- $\quad$ GPIO 0/GPIO 2 - General purpose I/O pins

- RST - RESET pin for the device

- GND- Ground

- $\quad$ CH_PD - Chip down Pin, 3.3V supply with 1K resistor

AT Commands of ESP 8266:

- AT: To start the wifi module

- $\mathrm{AT}+\mathrm{CWMODE}=1$ :

To set the mode of wifi

- AT+CIPSTA_DEF="IP

"GATEWAY", "SUBNET":

Address",

To set LAN connection

- $\mathrm{AT}+\mathrm{CWJAP}=$ "SSID", "PASSWORD”:

To connect with WIFI network

- $\mathrm{AT}+\mathrm{CIPMUX}=1$ :

To set channel

- $\quad \mathrm{AT}+\mathrm{CIPSTART}=\mathrm{TCP}$, "IP Address", PORT:

To connect with server

- $\mathrm{AT}+\mathrm{CIPSEND}=4$, Data Length:

To send data to server

- AT+CIFSR:

To check module Parameter (IP address, GATEWAY, SUBNET)

\subsection{Server}

In this project, we are using Websocket server which is a communication protocol. The WebSocket protocol is based on independent TCP protocol. The Websocket protocol makes communication between a client and server, providing the real-time data which is transfer from and to the server respectively. This allow messages to passed back while keeping connection open. In this way two way 
bidirectional communication can take place between a client and server. The communication in this project takes place over TCP port number 9000.

Client need to sends a WebSocket handshake request to establish a WebSocket connection and in response server will return a response.

\section{Client Request:}

GET /chat HTTP/1.1

Host: 178.16.1.22

upgrade: WebSocket

connection:Upgrade

Sec-webSocket-Key:x33JJhfnMmkD89kjM121=

Sec-websocket-Protocol: chat

Sec-webSocket-Version:13

origin: http://178.190.23.849

\section{Server Response:}

http/1.1 101 Switching Protocols

Upgrade: WebSocket

Connection: Upgrade

Sec-webSocket-Accept: Hsmjg10sdjLSH0Fgjkledgj3=

Sec-websocket

After a handshake response server and hardware are ready to send and receive the data bidirectionaly.Server will contain calculations for least count and alert count. Alert count will be calculated on the basis of $80 \%$ of main count.

$$
\text { Alert Count }=\text { Main count } * 80 \%
$$

Server contain a data base in which data from hardware will get stored. Similarly, data for hardware will also stored in database.

\section{LITERATURE SURVEY}

The research work is going on for collecting data packet (count) in real-time even in bad situations where there is poor network for receiving the data packet. In this project ESP-8266 WIFI module is used to send the data packet to server which work on frequency of 2.4 to $2.5 \mathrm{GHz}$. Hence with this project implementation we are giving alerts to the hardware when count limit is exceeded, also if the count keeps on exceeding final buzzer gets activated and it turn off the hardware. Also in case of server fail it detect the data packet of the machine where the count has occurred and sends count to server after re-connection with server.

Patel Urvija J. and Nehal G. Chitaliya[1]:This study investigates the effects to control and monitor the position and to send commands to person. Using Internet protocol operator can send the command from server to ESP module. And person will collect the information, so that he can work according to that.

S. Kathiresan and M. Janarthanan[2]: This project presents the monitoring and controlling the industrial parameter such as speed, torque, protection for single phase induction motor from overloading through Bluetooth. The system is design to maintain reliability, security and to solve many types of faults.

G.Prabhakar Reddy and M. Vijaya Lakshmi[3]: This paper presents the design of Internet of Things monitoring system for a safety measures for workers which is most essential in underground of mining areas. Project contain different types of sensors which are used to control and monitors the parameters of surrounding. All the data passes through ESP8266 WIFI module.

Mr. Krupal Patel, Mr. Jignesh Patoliya and Hitesh Patel[4]: This study investigate the different types of protocol used in IOT (Internet of Things) devices such as MQTT, TCP/IP protocol. This project is used TCP/IP protocol in which ESP8266 WIFI module will get the commands form server and output of wifi module is used for home automation.

\section{PROPOSED METHODOLOGY}

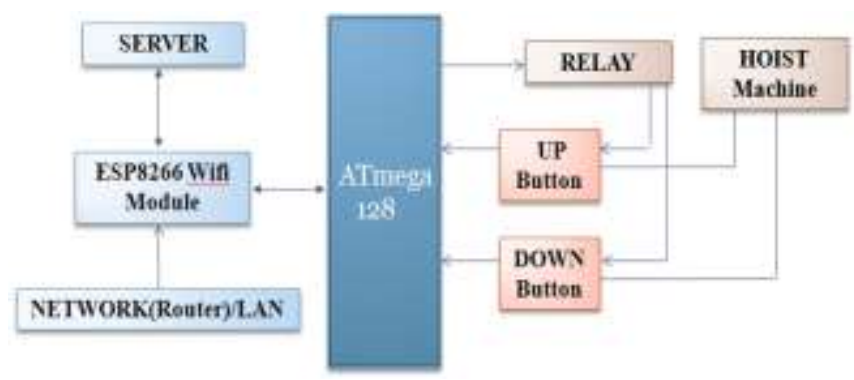

Fig -4: System flow

ATmega128 is heart of overall system where it is interfaced with ESP8266 wifi module which is bidirectional in working. Wifi module is connected to the Router which is used to initialize wireless transmission. Wifi module is connected to Server wirelessly which is used to transmit and retrieve the commands for hardware. Hardware passes its data packet to server via ESP8266 wifi module. On the other hand, Relay is output of controller to buttons which is used to enable the UP and Down buttons of Hoist system. By operating UP and DOWN buttons count will start to increase.

\section{WORKING}

Hardware section contain WIFI module which have a complete AT command library which is used for communication. This WIFI module is integrated with ATmega128 controller. Communication between WIFI module and controller is operating on serial communication. UART is a used to transmit and receive the commands to and from WIFI module and controller. Server is remote control for this project. Means Hardware will get each and every commands from server. To communicate with server, WIFI module need to ping to server for connection with server IP and port. Due to this connection, system will work as bidirectional. When hardware will ping to server then server will respond with the necessary data and depending on that data hardware will perform further operation. 
Every spare part has their life to run. The count will get increased by up and down button of hoist system. Machine count will be monitored by hardware. In running condition, hardware will send the count to server for the particular. Server will check the count from hardware and will compare with predefined life of server part. If hardware count reaches to $80 \%$ of predefined limit or count reaches to its alert zone, then hardware and server will generate alert. On alert generation buzzer will beep continuously after 15 seconds until hardware reaches to its main count. If spare part is in condition to run more than its predefined limit, then server will send additional count to hardware. Hardware will run till next additional count. When hardware reaches to new limit (additional count) then hardware will turn off the machine count and buzzer will beep continuously for 10 seconds. This indicates that the life of particular spare part is over and need to replace the with new. After replacing the part, server will again send next limit of another particular spare part which is addition of life of new spare part and last count from hardware and hardware will perform according to that. This process will run continuously for every spare part.

\section{CONCLUSION}

The Hoist system plays an important role in industries to lift weights. Due to this methodology industrial machine will get monitored and controlled. In point of reducing human errors to calculate the life of spare part of machine, design of such hardware is important. The proposed system gives real time monitoring, better accuracy, reliability in the industrial application.

\section{REFERENCES}

[1]. Patel Urvija J. and Nehal G. Chitaliya (2016)“IP Based Device Control and Monitoring System Using Raspberry Pi BasedOn IOT"International Journal for Innovations in Engineering and Technology, Volume 7 Issue 1 June.

[2]. S. Kathiresan and M. Janarthanan (2016), "Design and Implementation of Industrial Automation Using IOT and Bluetooth" International Journal for Advanced Research Trends in Engineering and Technology, Volume3, Issue 19, April.

[3]. G.Prabhakar Reddy and M. Vijaya Lakshmi (2015) "IOT in Mines for Safety and Efficient Monitoring"International Journal for Advanced Research in Computer Engineering \& Technology, Volume 4 Issue 11, November.

[4]. Mr. Krupal Patel, Mr. Jignesh Patoliya and Hitesh Patel(2015) "Low cost Home Automation with ESP8266 and Lightweight Protocol MQTT'Transactions on Engineering and Sciences. 